\title{
Analyzing flow anisotropies with excursion sets in relativistic heavy-ion collisions
}

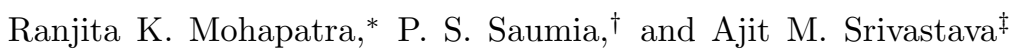 \\ Institute of Physics, Sachivalaya Marg, Bhubaneswar 751005, India
}

\begin{abstract}
We show that flow anisotropies in relativistic heavy-ion collisions can be analyzed using a certain technique of shape analysis of excursion sets recently proposed by us for CMBR fluctuations to investigate anisotropic expansion history of the universe. The technique analyzes shapes (sizes) of patches above (below) certain threshold value for transverse energy/particle number (the excursion sets) as a function of the azimuthal angle and rapidity. Modeling flow by imparting extra anisotropic momentum to the momentum distribution of particles from HIJING, we compare the resulting distributions for excursion sets at two different azimuthal angles. Angles with maximum difference in the two distributions identify the event plane, and the magnitude of difference in the two distributions relates to the magnitude of momentum anisotropy, i.e. elliptic flow.
\end{abstract}

PACS numbers: 25.75.-q,12.38.Mh,98.80.Es

Analysis of anisotropies of flow in relativistic heavy-ion collision experiments (RHICE) has provided valuable information regarding the properties of matter produced during early transient stages in these collisions [1]. Early investigations focused on the elliptic flow relating to the 2nd Fourier coefficient of momentum anisotropy as a function of azimuthal angle, as well as couple of other flow coefficients. Drawing correspondence of the underlying physics of fluctuations between RHICE and the Cosmic Microwave Background Radiation (CMBR) anisotropies, it was pointed out by us in ref. 2] that a wealth of information is contained in plots of root-mean-square values of flow coefficients $v_{n}^{r m s}$ for a large range of values of $n$, from 1 to 30-40. It was also proposed in 2] that the presence of superhorizon fluctuations can lead to the suppression of superhorizon modes, as well as the existence of acoustic oscillations in plots of $v_{n}^{r m s}$ in RHICE, similar to the celebrated acoustic peaks of CMBR arising from inflationary density fluctuations. This suppression of superhorizon modes in RHICE may have been seen in the analysis reported by Sorenson for RHIC data [3].

This very useful interplay of physics from CMBR to RHICE was further explored in ref. [4] following the effects of magnetic field on the power spectrum of CMBR anisotropies [5]. Utilizing that insight, it was proposed in [4] that presence of strong magnetic field in the initial stages of a non-central collision in RHICE can affect flow anisotropies, for example, it can lead to enhancement of flow coefficients. In particular, it could lead to enhancement in the elliptic flow coefficient $v_{2}$ by almost $30 \%$ (which may allow for a higher value of viscosity). A different analysis in ref. [6] in the strong field limit further explored these effects and gave similar results for the enhancement of $v_{2}$.

In this paper we explore another common feature between the analysis of CMBR and the flow analysis in RHICE. This is based on a technique recently proposed by us for investigating any anisotropic expansion stage in the history of the universe by analyzing the shapes of patches of CMBR fluctuations (the excursion sets) at the surface of last scattering [7]. The main idea underlying this analysis [7] was the following. If the density perturbations generated initially are statistically isotropic, then they will get deformed in a specific direction if the universe ever went through an anisotropic expansion. This (statistically averaged) deformation can be detected by a shape analysis of fluctuation patches of CMBR. An anisotropic expansion of the universe has very strong similarities with the anisotropic flow expected in RHICE. The situation of anisotropic expansion of the universe is directly realized in the form of elliptic flow for non-central events. Flow anisotropies are generally present in every event in RHICE and all values of $v_{n}^{r m s}$ are non-zero in general due to the presence of fluctuations of different scales even in the central collisions 2]. This suggests that the shape analysis of excursion sets used in ref. 7] should provide useful information for flow anisotropies in RHICE as well, especially for the elliptic flow. We will see below that this is indeed true. We consider fluctuations of transverse energy or particle number as a function of the azimuthal angle and rapidity. This is like CMBR fluctuations at the last scattering surface. Then by considering fluctuations above/below a certain threshold value, we generate the excursion sets of fluctuations, we call them simply as fluctuation patches. To see the possibility of detecting anisotropic flow, we model flow by imparting extra anisotropic momentum to the momentum distribution of particles obtained from HIJING [8]. We then analyze the shape/size distribution of the fluctuation patches at two different azimuthal angle. We will see that azimuthal angles with maximum difference in the two distributions identify the event plane, and the magnitude of difference relates to the magnitude of momentum anisotropy, i.e. elliptic flow.

\footnotetext{
*Electronic address: ranjita@iopb.res.in

${ }^{\dagger}$ Electronic address: saumia@iopb.res.in

‡Electronic address: ajit@iopb.res.in
} 


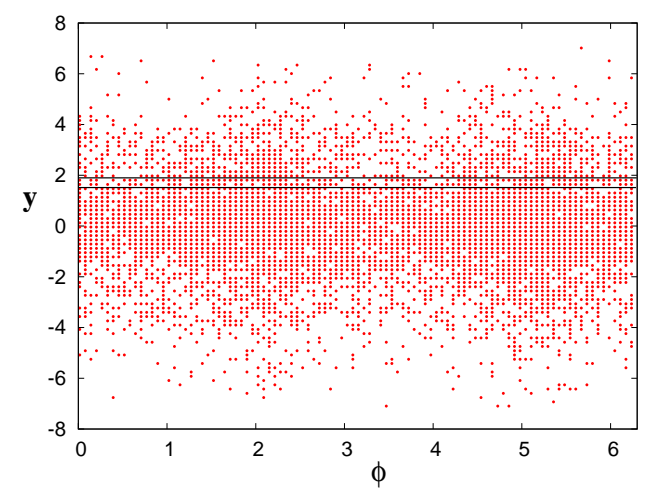

FIG. 1:

Plot of excursion sets (fluctuation patches) in the azimuthal angle $\phi$ (in radians) and the rapidity $y$ space. Horizontal solid lines show the slicing used for widths of filled patches.

For analyzing anisotropic expansion history of the universe in 7], we had examined the size distribution of patches on the surface of last scattering (which is a two sphere $S^{2}$ ) along $\theta$ and $\phi$ directions. This will be different for RHICE. Since our interest is in probing flow anisotropy, say the elliptic flow in a non-central event, we would like to compare the shape/size distribution of fluctuations along the $\mathrm{X}$ axis with that along the $\mathrm{Y}$ axis, where the $\mathrm{X}$ and the $\mathrm{Y}$ axis correspond to the event plane. As we mentioned, we model anisotropic flow in HIJING by multiplying a factor $f_{p_{x}}$ to the momentum $P_{x}$ of every final state particle. For central events $f_{p_{x}}$ is taken to be 1 while for non-central events $f_{p_{x}}$ is taken to vary from 1.1 to 1.4 representing the momentum anisotropy resulting from elliptic flow. Precise value of momentum anisotropy represented by $f_{p_{x}}$ is not an issue here (though the values used here are of right order of magnitude, say, $f_{p_{x}}=1.2$ implying about $20 \%$ anisotropy). Our intention is only to show the systematic pattern in which the difference between the size distributions of excursion sets at different azimuthal angle probe the event plane and strength of elliptic flow. A quantitative correspondence can only be established by incorporating our analysis in hydrodynamical simulations and we hope to report such an analysis in a future work.

With the (anisotropic) momentum distribution of particles from HIJING, we collect particle numbers as well as transverse energy in different $\phi$ and $y$ bins where $\phi$ is the azimuthal angle in the event plane and $y$ is the rapidity. We use 100 bins for full $2 \pi$ range of $\phi$ and 100 bins for the full range of $y$. We have also considered smaller range of rapidity $y$ and the results are similar. Full range of $y$ gives better statistics. To exclude very large values of $y$ we use a lower cutoff for $P_{T} \simeq 150 \mathrm{MeV}$. For each event thus we get the particle number/transverse energy as a function of $\phi$ and $y$. By subtracting the average values of these quantities we get particle number fluctuations/transverse energy fluctuations as a function of $\phi$ and $\eta$. We then consider a threshold value for these fluctuations and consider only those $\phi, y$ bins where particle number or transverse energy fluctuations is above (or below) this threshold value. This provides us with the excursion sets for the relevant fluctuations. Fig.1 shows the excursion sets for transverse energy fluctuations obtained from HIJING for a single $\mathrm{Au}$-Au collision event at $\sqrt{s_{N N}}=2 \mathrm{TeV}$. (Here, we will be presenting results for transverse energy fluctuations. results for number fluctuations are very similar to these, hence we do not discuss them separately.) We consider here non-central events with the impact parameter lying between $b=3-4$ $\mathrm{fm}$. Here we have used $f_{p_{x}}=1.2$ (for modification of the momenta of particles from HIJING) and fluctuations above $0.2 \times$ maximum fluctuation have been included to generate the excursion sets. We use here large collision energy as relevant for LHC. The size distributions for small number of particles have large statistical errors. For the case of RHIC energies one will need to combine much larger number of events to control statistical errors.

What we want to know is the average width of the fluctuations along $\phi$ direction near $\phi=0$ and compare it with the similar average width near $\phi=\pi / 2$. This will probe the elliptic flow. (Clearly, the same result will be obtained by comparing distributions at $\phi=\pi$ and $\phi=3 \pi / 2$.) We determine the distribution of widths along the $\phi$ direction in a $45^{\circ}$ wide arc centered at $\phi=0$ and compare it with the distribution of widths (again, in a $45^{\circ}$ wide arc) centered at $\phi=\pi / 2$. To calculate these distributions we proceed as follows. We divide the entire region shown in Fig.1 (with $y$ varying within full range, and $\phi$ ranging from 0 to $360^{\circ}$ ) into thin slices (with a rapidity width of 0.18 ) along the $\phi$ direction, as shown by horizontal lines in Fig.1. Using these slices, we determine the $\phi$ widths of various filled patches. We then plot the frequency distributions (histograms) of the widths of the intersections of all the patches with these slices at $\phi=0$ and at $\phi=\pi / 2$ (within $45^{\circ}$ arcs). For the isotropic expansion case, without any flow anisotropy, we expect these two histograms to almost overlap. For anisotropic expansion case we expect that fluctuation patches will be stretched more at $\phi=\pi / 2$ than at $\phi=0$, as system expands faster along $\phi=0$ (and $\phi=\pi$ ) directions for 

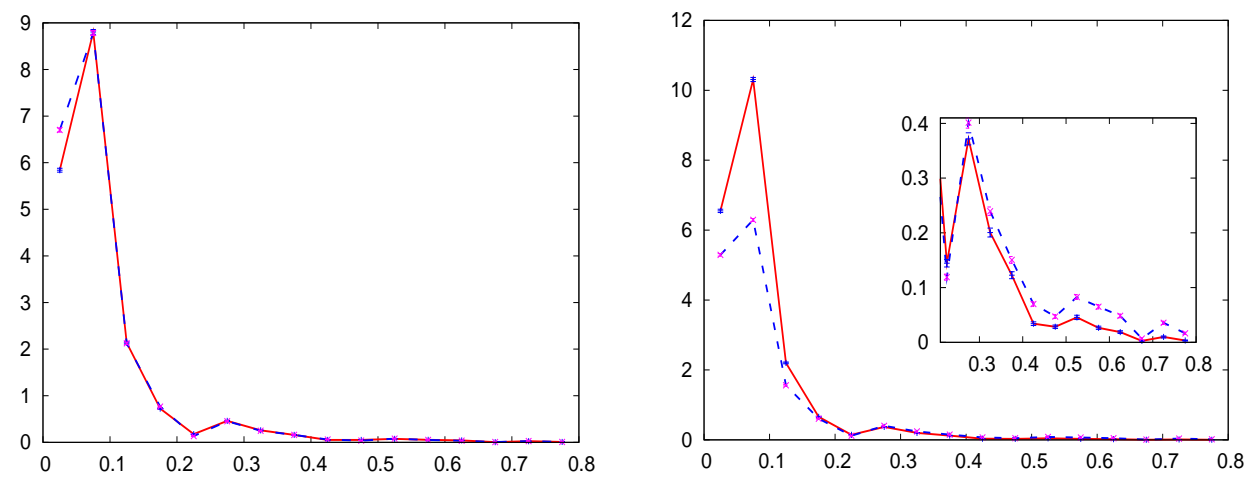

FIG. 2:

(a) Plots of the histograms of the widths (in radians) of filled patches for $f_{p_{x}}=1$. Smooth curves join the points which are marked with corresponding $(\sqrt{N})$ error bars. Distribution for widths at $\phi=0(\phi=\pi / 2)$ are shown by solid (dashed) plots.

(b) Plots for the case with momentum modification, with $f_{p_{x}}=1.2$. The inset in (b) shows the plots for larger widths.

a non-central collision. Any relative difference, between the two histograms will, therefore, imply the presence of an anisotropy of expansion (apart from the possibility of any initial anisotropy, we will discuss it below).

Fig. 2a shows the frequency distributions (histograms) of the widths of the intersections of the slices with the filled patches (excursion sets) at $\phi=0$ (solid curve) and $\phi=\pi / 2$ (dashed curve) for the case with $f_{p_{x}}=1$ (i.e. no momentum anisotropy. The horizontal axis corresponds to the widths of the slices (in radians) along $\phi$, with histogram bin having width of 0.05 radians. The vertical axis gives the frequency $N$ of the occurrence of the respective widths in all the slicings of excursion sets (such as in Fig.1, but now for $f_{p_{x}}=1$ ). The error bars denote the statistical uncertainty of $\sqrt{N}$ for the frequency $N$ in each bin. As we are comparing distributions for two different data sets (one at $\phi=0$, the other at $\phi=\pi / 2$ ), we normalize the distributions, as well as the errors, with the total number of particles included for each data set. We combine histograms of 500 events in HIJING with the X axis of each event (with same event parameters such as the impact parameter, collision energy etc.) representing the minor axis of the ellipse representing the overlap of the two colliding nuclei. We can see from Fig.2a that the two histograms, are almost overlapping. This is interesting as we are considering here non-central collisions (with $b=3-4 \mathrm{fm}$ ). This shows that the momentum distributions and all fluctuations obtained from HIJING (without any momentum modification, i.e. $f_{p_{x}}=1$ ) are isotropic. Any anisotropy will result from effects of particle interactions, primarily from collective flow effects, and we will represent it by using values of $f_{p_{x}}$ different from 1 . We mention here that the peak near 0.1 radians in Fig. 2 will represent fluctuations of size order $1 \mathrm{fm}$ at the distance of about $10 \mathrm{fm}$ representing the size of initial nuclei. As discussed in ref.[2], initial fluctuations are expected because of the nucleon size of order $1 \mathrm{fm}$ (as well as quantum fluctuations in nucleon coordinates.) Such a peak thus may have useful information about the scale of fluctuations in the early stages of system evolution.

We now consider an anisotropic momentum distribution by taking $f_{p_{x}}=1.2$ to modify particle momentum distribution from HIJING. (Note that this is just to model the effects of flow.) Fig.2b shows the plots of distributions of widths for this case. We see that the distribution at $\phi=\pi / 2$ is smaller than the distribution at $\phi=0$ (beyond the error bars) for small widths while the situation is reversed for larger widths (as shown by the inset in Fig.2b). This is expected as the stretching of fluctuation patches will be more significant at $\phi=\pi / 2$ due to elliptic flow. Stretching makes fluctuation patches larger, hence the distribution for large widths is higher for $\phi=\pi / 2$ than for $\phi=0$. This is suitably compensated at smaller angles where $\phi=\pi / 2$ distribution falls below the $\phi=0$ distribution. We find that the ratio of the heights of the two peaks is directly related to the value of $f_{p_{x}}$ representing the effects of elliptic flow. For $f_{p_{x}}=1.1,1.2,1.3$, we find this ratio to be 1.3, 1.6, 2.0. Thus, the magnitude of elliptic flow can be probed directly with the comparison of the distributions of widths of fluctuation patches at $\phi=0$ and at $\phi=\pi / 2$. Note that our modeling of flow in terms of a factor like $f_{p_{x}}$ is ad hoc. Thus we do not try to derive any functional relationship between this factor and the ratios of the heights of the two peaks above (or try to give error bars for these etc.). Our purpose here is to show specific patterns of these distributions depending on momentum anisotropy. We also mention here that in actual hydrodynamic flow, the effect of flow on the momentum of particles will depend on the $\mathrm{Y}$ coordinate of the particle inside the plasma (in the event plane), being less important for larger Y values, as well as on the distance from the center. To more accurately represent the $Y$ dependence of flow anisotropy, we have compared the $\phi=0$ distribution using $f_{p_{x}}=1.3$ with the $\phi=\pi / 2$ distribution with $f_{p_{x}}=1.1$. The difference between the two distributions is found to be closer to the difference obtained for the constant value of $f_{p_{x}}=1.2$ for both the 

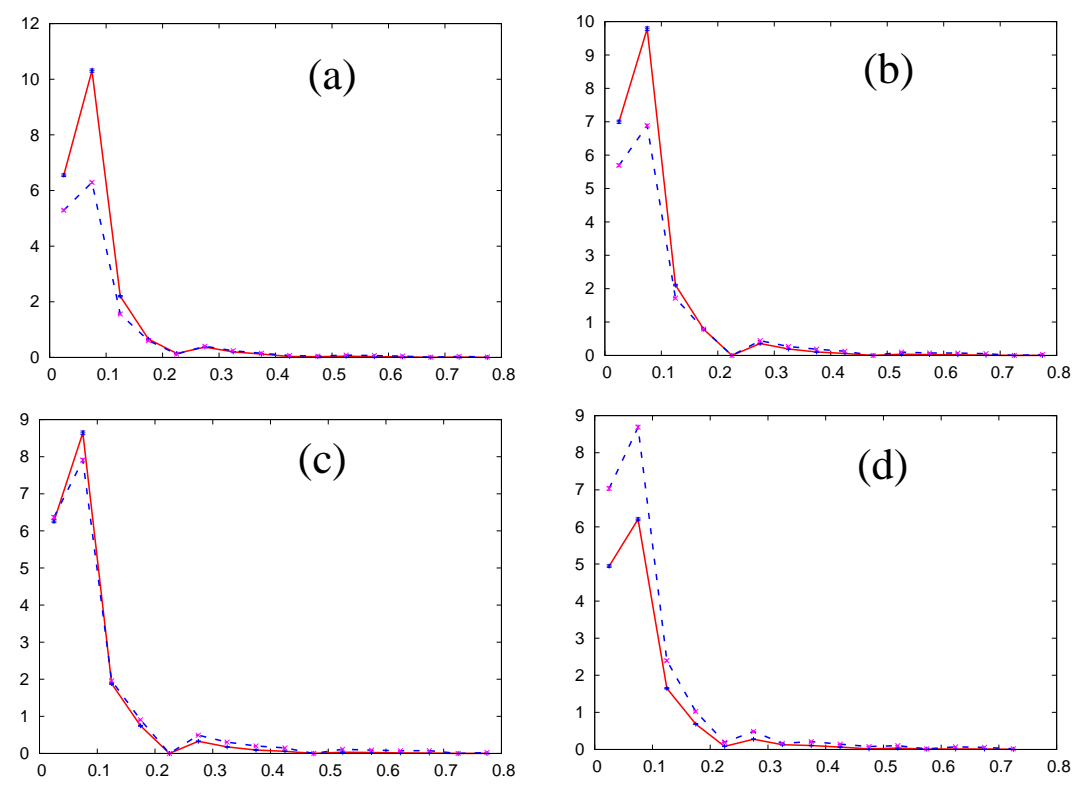

FIG. 3:

Plots of histograms (for 500 events each) at $\phi=\phi_{0}$ (solid curve) and at $\phi_{0}+\pi / 2$ (dashed curve) for $\phi_{0}=0$ (a), $\pi / 16$ (b), $\pi / 8$ (c), and $\pi / 4(\mathrm{~d})$.

distributions. Since we are only interested in comparative changes in the two distributions, for simplicity, we will continue to use a constant value of $f_{p_{x}}$ for determining the difference between the $\phi=0$ and $\phi=\pi / 2$ distributions.

Of course this requires identification of the event plane so that statistics of a large number of events can be added up for controlled statistical errors. We now show that our technique can provide a new way to identify the event plane. In Fig. 3 , we show a series of plots of widths distributions at $\phi=\phi_{0}$ (solid plot) and at $\phi=\phi_{0}+\pi / 2$ (dashed curve) for the angle $\phi_{0}=0, \pi / 16, \pi / 8, \pi / 4$. We note that the ratio of the two peaks (solid curve and dashed curve) is largest for $\phi_{0}=0$ which represents the event plane. Thus, one needs to simply plot the two width distributions at angles $\pi / 2$ apart and keep changing the initial angle until the two distributions have maximum difference. This will identify the event plane. (Though we note that maximum overlap of the two distributions seems to happen at $\phi_{0}=\pi / 8$ and not at $\pi / 4$ as one would have expected. This could be due to the large arc size of $45^{\circ}$ used for calculating the individual distributions.) We have also compared the two distributions by varying the separation of $\phi$ at which they are evaluated. When the separation is larger than about 50-60 the difference becomes almost similar up to $90^{\circ}$ separation. With the physics of ellptic flow in view, we choose $90^{\circ}$ separated distributions for comparison. Fig.3 is shown with collection of data from 500 events each. One will need to do this on event-by-event basis to be able to identify the event plane in each case. Fig.4 shows the same plots as in Fig.3, but now for single events. We see that statistical errors are larger. For larger collision energies, statistical errors will be under better control. Also, one may arrive at the event plane by an iterative process. First one can roughly identify event planes for each event (as in Fig.4). Then a large number of events can be combined for each choice of $\phi_{0}$ to maximize the difference between the two plots. This can be done by systematically changing the event plane for each event, until the all-event-sum histograms (as in Fig.3) lead to maximum difference between the two histograms.

In this paper we have shown that a shape/size analysis of excursion sets can lead to a completely new way of identifying the event plane in RHICE and to quantify the magnitude of flow anisotropies. This method is novel, and completely independent of other conventional methods. This technique can, thus, be used to cross-check the results obtained by other conventional techniques. Further, the entire distribution of sizes of excursion sets, as shown in Fig.2, will contain valuable information about nature of fluctuations and that of anisotropic flow. Also the width distribution along the rapidity should provide information about the longitudinal scaling of fluctuations. These issues can be probed using hydrodynamic simulations. Work is underway along these line and we hope to present it in future. We mention here that anisotropic expansion of the universe was discussed in ref. 7] using Fourier transform technique as well as direct shape analysis of excursion sets. In this paper we have used the direct shape analysis method for investigating flow anisotropy. It will be useful to also use Fourier transform technique for this purpose. Note that this technique will detect any effects present in the system which lead to anisotropy of momenta. For example the presence of initial strong magnetic field (as well as induced electric field) will lead to anisotropies [4, 6] and should 

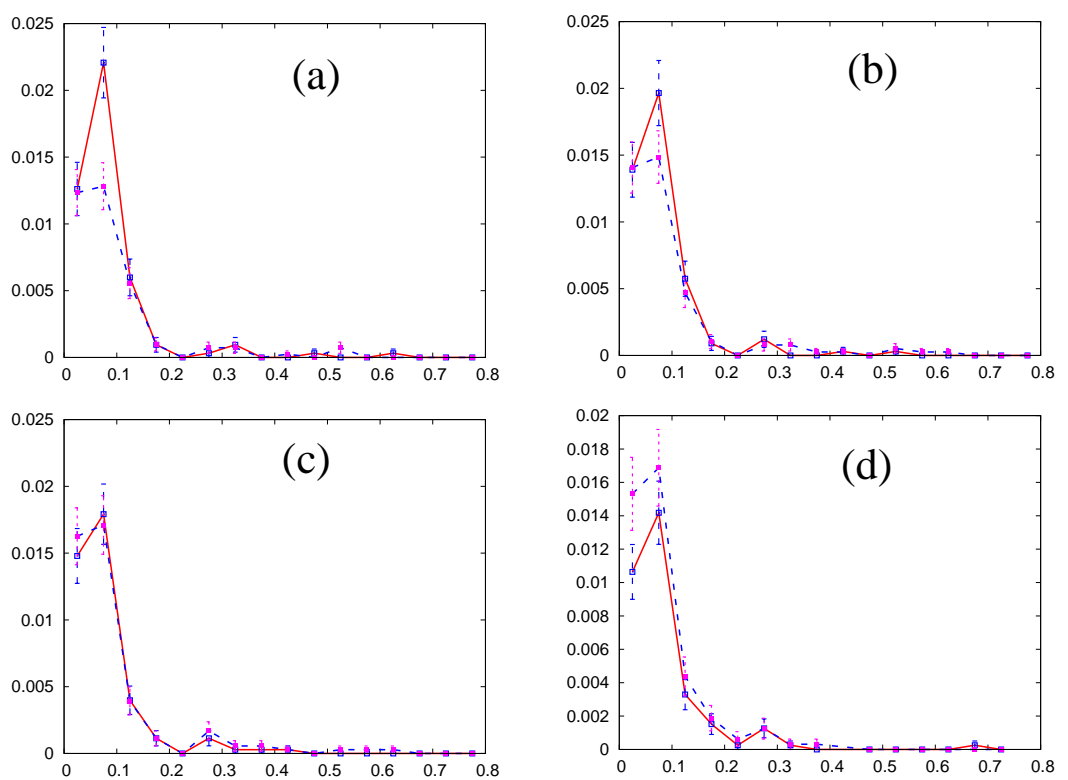

FIG. 4:

Series of plots as in Fig.3, for single event case each.

leave imprints on these distributions of widths. Even the presence of any initial anisotropies of fluctuations, such as for deformed nuclei, could be detected in this method. Important issue then will be to distinguish any initial anisotropy (as from a deformed nucleus) from anisotropy resulting from anisotropic flow. In this respect this technique of direct shape analysis seems particularly effective, in comparison to, e.g. the Fourier transform technique, as discussed in detail in ref. 7]. We hope to address these issues in a future work. We point out that other methods such as Minkowski fucntionals [9], analysis of genus statistics of excursion sets [10] etc., have also been very effectively used for analyzing morphology of fluctuations in CMBR as well as in large scale structure in the universe, and it will be fruitful to apply these techniques also for analyzing the nature of fluctuations as well as flow anisotropies in RHICE.

We are very grateful to Abhishek Atreya, Partha Bagchi, and Anjishnu Sarkar for useful discussions and comments.

[1] J.-Y. Ollitrault, Phys. Rev. D 46, 229 (1992); S. Voloshin and Y. Zhang, Z. Physik C70, 665 (1996); S.A. Volosin, A.M. Poskanzer, and R. Snellings, arXiv:0809.2949.

[2] A.P. Mishra, R. K. Mohapatra, P. S. Saumia, and A. M. Srivastava, Phys. Rev. C 77, 064902 (2008); Phys. Rev. C 81, 034903 (2010).

[3] P. Sorensen, Proc. 24th Winter Workshop on Nuclear Dynamics, 2008, arXiv:0808.0503.

[4] R. K. Mohapatra, P. S. Saumia, and A. M. Srivastava, Mod. Phys. Lett. A26 2477 (2011).

[5] J. Adams, U.H. Danielsson, D. Grasso, and H. Rubinstein, Phys. Lett. B 388, 253 (1996).

[6] K. Tuchin, arXiv:1108.4394.

[7] R. K. Mohapatra, P. S. Saumia, and A.M. Srivastava, arXiv:1111.2722,

[8] M. Gyulassy, D. H. Rischke, and B. Zhang, Nucl. Phys. A 613, 397 (1997).

[9] M. Migliaccio et al. Nucl. Phys. B194(Proc. Suppl.) 278 (2009); J.V. Sheth and V. Sahni. astro-ph/0502105.

[10] G. Rossi, P. Chingangbam, C. Park, arXiv:1003.0272. 